Title : will be set by the publisher

Editors : will be set by the publisher

EAS Publications Series, Vol. ?, 2018

\title{
STABILITY AND EVOLUTION OF SUPER-MASSIVE STARS (SMS)
}

\author{
A. Just ${ }^{1}$ and P. Amaro-Seoane ${ }^{1}$
}

\begin{abstract}
Highly condensed gaseous objects with masses larger than $5 \cdot 10^{4} M_{\odot}$ are called super-massive stars. They are thought to be possible precursors of super-massive black holes in the centres of galaxies. In the quasistationary contraction phase, the hydrostatic equilibrium is determined by radiation pressure and gravitation. The global structure is that of an $n=3$ polytrope at the stability limit. Small relativistic corrections for example can initiate a free fall collapse due to the 'post Newtonian' instability. Since the outcome of the final collapse - A super-massive black hole or hypernova - depends sensitively on the structure and the size of the object, when the instability sets in, it is important to investigate in more detail the contraction phase of the SMS. If the gaseous object is embedded in a dense stellar system, the central star cluster, the interaction and coupling of both components due to dynamical friction changes the energy balance and evolution of the SMS dramatically. Dynamical friction between stars and gas, which can be estimated semi-analytically (see Just et al. 1986), has three different effects on the two-component system: 1) The gas is heated by decelerating the stars. This may stall the contraction process for a while until the stars in the 'loss cone', these which cross the SMS, lost their kinetic energy (for the total heating rate see Amaro-Seoane \& Spurzem 2001). 2) This cooling of the loss cone stars lead to a mass segregation in the stellar component resulting in a much more condensed central stellar core. 3) The inhomogeneities due to the gravitational wakes in the gas changes the effective absorption coefficient of the gas. This affects the condition for hydrostatic equilibrium and may give essential deviations from the $n=3$ polytrope. We discuss in which evolutionary stages and parameter range these interaction processes are relevant and how they can influence the stability and evolution of the SMS.
\end{abstract}

\footnotetext{
${ }^{1}$ Astronomisches Rechen-Institut, Mönchhofstraße 12-14 D-69120 Heidelberg, Germany; e-mail: just@ari.uni-heidelberg.de \& pau@ari.uni-heidelberg.de
}

(c) EDP Sciences 2018

DOI: (will be inserted later) 
Title : will be set by the publisher

\section{Introduction}

In the standard picture of galactic structure, most galaxies harbour a central Super-massive Black Hole (SMBH), which is quiet in normal galaxies and responsible for quasar and Seyfert activities in active galaxies. But the building process of SMBHs is still not fully understood. Is the way of creating a SMBH with more than $10^{6} M_{\odot}$ a direct collapse of a gas cloud, is it the growth of a small seed black hole in a dense star cluster, or is it the remnant of a hypernova of a super massive star like object (SMS)?

In the building process of a SMBH two basic paths can be distinguished (Rees 1984). At the stellar dynamical path the gas cloud initially forms a massive central star cluster or bulge. Stellar and dynamical evolution including star-star collisions than form a seed black hole with initial mass up to $10^{4} M_{\odot}$. Subsequently this black hole grows by accretion of gas and stars. Here the basic problem of the growth is the feeding rate. Only a very small fraction of stars have highly excentric orbits to reach the tidal radius of the black hole and refilling this 'loss-cone' is a complicated matter (for details see Amaro-Seoane \& Spurzem 2001] and references therein). The gas dynamical path starts also with a collapsing gas cloud, but here the cloud collapses directly to a highly condensed gas ball. The lifetime of this super-massive object is very short (see section 2) such that star formation in this condensed phase does not occur. The dynamical collapse can go on to end up with a SMBH, or it can reach a hydrostatic state, which than contracts by radiative cooling. We call a gas ball with mass $M>5 \cdot 10^{4} M_{\odot}$ in hydrostatic equilibrium a super-massive star (SMS). It is not proofed that a direct collapse is possible, because explosive hydrogen burning would destroy more or less the whole object. Even the final collapse of a SMS, which becomes unstable due to the post-Newtonian instability, will be stopped by hydrogen burning (Fuller et al. 1986).

In a more realistic scenario of galaxy formation, a central bulge or dense star cluster will form first, and at a later stage there will occur strong gas infall initiated by a galaxy merging process or a bar instability. In this case the super-massive gaseous object will not be isolated and interaction processes with the star cluster can change the evolution dramatically. Hara (1978) showed in an analytic approximation, that heating of the SMS by drag forces of the stars crossing the SMS can stop the contraction for some time. In a numerical simulation Langbein et al. (1990) showed that the energy input due to star-star collisions can dominate over heating by drag forces and leads to a rebounce of the SMS.

In the next section we give an overview over the properties of a SMS and in section 3 we discuss the interaction processes of the SMS with the surrounding star cluster in a more complete way. 


\section{The standard SMS}

In a SMS radiation pressure $P_{\text {rad }}$ dominates strongly over thermal pressure $P_{g}$, the fraction of which is

$$
\beta=\frac{P_{g}}{P_{g}+P_{\text {rad }}}=7.8 \cdot 10^{-4} M_{8}^{-1 / 2}
$$

where $M_{8}$ is the mass in units of $10^{8} M_{\odot} . \beta$ is independent of radius $\left(R_{p c}\right.$ given in pc) and does not change during contraction of the SMS. For $\beta<<1$ the structure of the SMS is very near to a $\mathrm{n}=3$ polytrope, which is the stability limit with zero total energy. The SMS is determined by mass $M$ and radius $R$. The central temperature $T_{c}$, central density $\rho_{c}$, gravitational and total energy $\left(E_{g}\right.$ and $\left.E_{t o t}\right)$ are given by

$$
\begin{aligned}
T_{c} & =1.9 \cdot 10^{4} K M_{8}^{1 / 2} R_{p c}^{-1} \\
\rho_{c} & =54 \bar{\rho}=10^{-13} \frac{g}{c m^{3}} M_{8} R_{p c}^{-1}=1.5 \cdot 10^{9} \frac{M_{\odot}}{p c^{3}} M_{8} R_{p c}^{-1} \\
E_{g} & =-\frac{3}{2} \frac{G M^{2}}{R}=-1.3 \cdot 10^{57} \operatorname{erg~} M_{8}^{2} R_{p c}^{-1} \\
E_{t o t} & =\frac{3}{2} \beta E_{g}=-1.5 \cdot 10^{54} \operatorname{erg~} M_{8}^{3 / 2} R_{p c}^{-1},
\end{aligned}
$$

respectively. The contraction rate of the SMS is determined by the Eddington luminosity

$$
L_{E d}=1.3 \cdot 10^{46} \frac{\mathrm{erg}}{\mathrm{s}} M_{8}
$$

leading to a contraction time

$$
t_{K H}=\frac{-E_{t o t}}{L_{E d}}=3.7 y r M_{8}^{1 / 2} R_{p c}^{-1}
$$

In parameter space (the $R, M$-plane), the regime of hydrostatic, stable equilibrium (where a SMS can exist) is bounded at the high radius end (at $R_{f f}$ ) where the Kelvin-Helmholtz time $t_{K H}$ (contraction time) equals the free fall time. At the low radius end it is bounded by the post-Newtonian instability $\left(R_{P N}\right)$ or for lower masses when nuclear burning at the centre sets in $\left(R_{n b}\right)$. In figure 1 the relevant regime for a SMS is shown. The Schwarzschild radius $R_{S}$ is far below the living region of a SMS. The evolutionary tracks are horizontal lines from right to left. The contraction time is also plotted showing that without re-heating the lifetime of the SMS is at most $2 \cdot 10^{4} \mathrm{yr}$ before nuclear burning or the post-Newtonian collapse sets in.

\section{SMS in a central star cluster}

In the central region of young galaxies with SMS both, stellar and gas densities, are very high exceeding $10^{8} M_{\odot} p c^{-3}$. Therefore the interaction rate of stars is very 


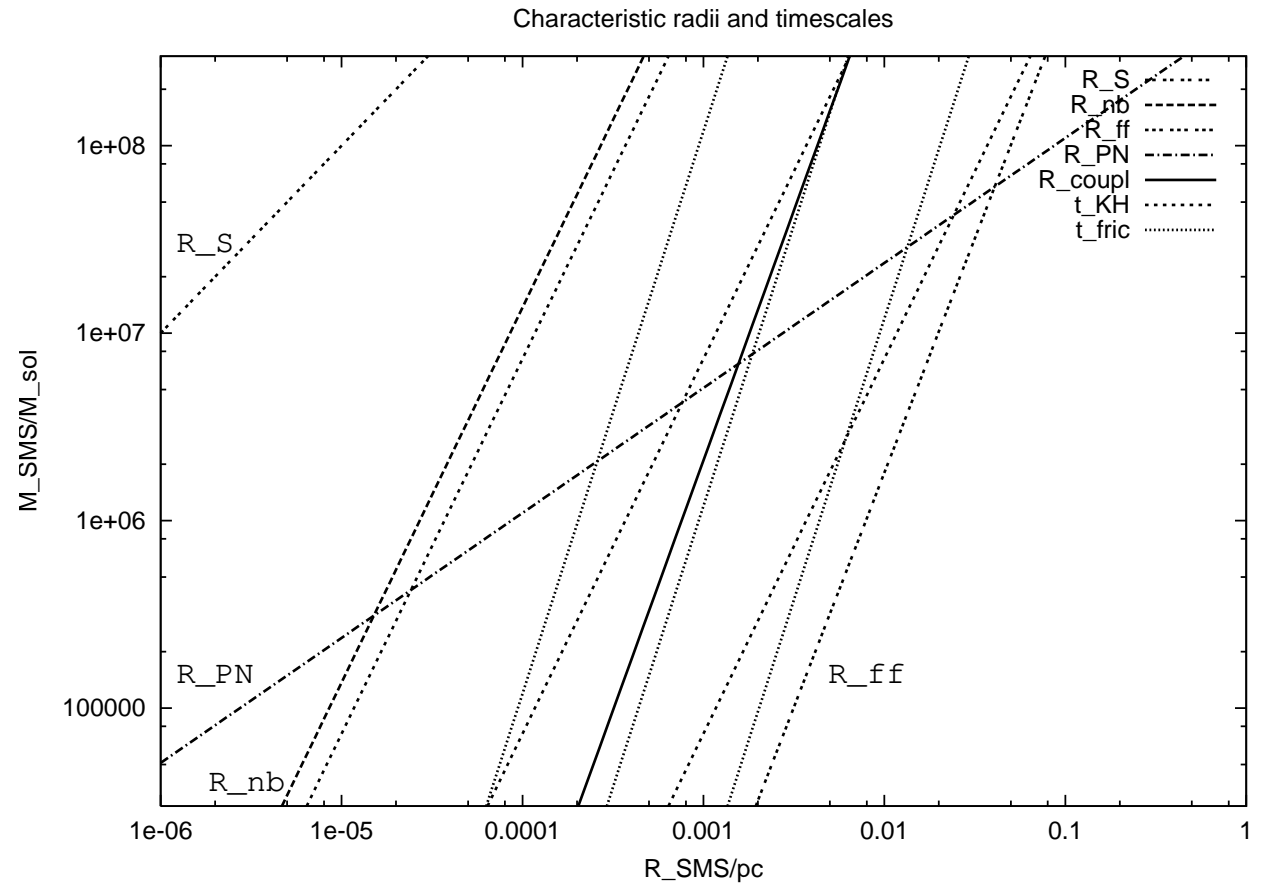

Fig. 1. The regime of hydrostatic contraction of a SMS bounded by $R_{f f}, R_{n b}$, and $R_{P N}$ is shown. For comparison the Schwarzschild radius $R_{S}$ is also plotted. Lines of constant contraction time $\left(t_{K H}=10^{2}, 10^{3}, 10^{4} \mathrm{yr}\right.$ from right to left) and of constant friction timescales $\left(t_{\text {fric }}=10^{1}, 10^{3}, 10^{5} \mathrm{yr}\right.$ from left to right) show that the efficiency of dynamical friction grows quickly during contraction. The regime, where the friction timescale is shorter than the contraction time, $R<R_{\text {coupl }}$, is also shown. For the stars a typical velocity of $5000 \mathrm{~km} / \mathrm{s}$ is used. For the friction timescale we assumed that the stars move only $1 \%$ of their orbital time through the SMS due to the high excentricity. Stars on more circular orbits are decelerated faster.

high and drag forces of 'loss-cone' stars (this are the stars crossing the SMS) can drive the evolution considerably. Earlier investigations (Hara 1978, Langbein et al. (1990) show that the timescale for dynamical coupling of the stellar system with the gas and for heating by stellar collisions is comparable or even shorter than the lifetime of a SMS.

Here we concentrate on the star-gas interaction and discuss the effects on the structure and evolution of the SMS. Stars moving through the gas produce density and velocity perturbations by gravitational forces and the geometrical cross section. The consequences of these local perturbations on the global behaviour of the SMS can be consistently estimated using a quasi-linear perturbation theory. In this method, all physical quantities are decomposed into a local mean value and a perturbation ( $\rho=\rho_{0}+\rho_{1}$ for the gas density e.g.). Then the time 
dependent perturbations are computed from the set of linearised equations and finally the quasistationary fluctuation field of the nonlinear quantities as functions of the mean quantities are derived by taking the statistical average over all stars. Dynamical friction between stars and gas is given by $\left\langle\rho_{1} \nabla \phi_{1}\right\rangle$ with gravitational potential $\phi$ (see Just et al. 1986). The level of approximation is similar to that of using Chandrasekhars dynamical friction formula. The advantage of the fluctuation theory is that the turbulent velocity field, corrections to the equation of state and to radiation transfer can all be derived consistently. It is also straightforward to include all relevant physical processes in the gas like radiation pressure, heating and cooling processes, viscosity or even magnetic fields.

The structure of the SMS is determined by hydrostatic equilibrium and a stationary radiation field in the diffusion limit. These assumptions are not automatically valid for the perturbations, because here the length and timescales are much shorter. The perturbations are time dependent and it depends on the scale, whether the radiation field will be compressed also. The character of the perturbations will be between the two extremes:

Isothermal In small scale fluctuations the inhomogeneities of the radiation field diffuse quickly away. Since the temperature is determined by the radiation, the compression of the gas is isothermal and the sound speed relevant for determining the Mach number of the stellar motion is the usual isothermal sound speed $\left(c_{s} \approx 100 \mathrm{~km} / \mathrm{s}\right.$ for a million solar mass SMS with radius $R=$ $0.01 p c)$. Most stars are highly supersonic in this case.

Adiabatic Larger fluctuations, where the radiation field is also compressed, are adiabatic. The Mach number is a factor of $\beta^{1 / 2}$ smaller than in the isothermal case. It is determined by the total pressure leading to typical Mach numbers of order unity for the stars.

The resultant density and velocity fluctuations of the gas and the variations in the radiation field change the mean local properties of the SMS systematically. In the SMS basically three different effects of the fluctuations can be important:

Dynamical heating The energy loss of the stars by dynamical friction heats the SMS in the inner region. The heating rate depends on the relative masses and densities of the star cluster and the SMS, and of the filling factor of the loss cone, which is a complicated matter to estimate. If the heating rate exceeds the cooling of the SMS, then the contraction can be stalled for a while.

Mass segregation Dynamical friction leads also to mass segregation of the stars. Loss cone stars become confined to the SMS. This happens, if the friction timescale $t_{\text {fric }}$ becomes smaller than the contraction time $t_{K H}$. In figure an estimation of $t_{\text {fric }}$ is shown and also the coupling radius $R_{\text {couple }}$ of the SMS for stars with an initial velocity of $5000 \mathrm{~km} / \mathrm{s}$. In more compact SMS loss-cone stars are effectively stopped by dynamical friction. Since dynamical friction has its maximum just above the sound speed, confined stars will be 
decelerated quickly below the sound speed building a new highly condensed stellar core. Then the question is, whether this new core can decouple from the surrounding to build a new smaller SMS-star cluster system with higher density.

Equation of state The turbulent velocity field and non-isothermal density fluctuations change the relative contribution of radiation pressure. This results in a different mean $\bar{\beta}$ of the form

$$
\bar{\beta}=\beta_{0}\left(1+C \frac{\left\langle\rho_{1}^{2}\right\rangle}{\rho_{0}^{2}}\right)>\beta_{0}
$$

which stabilises the SMS. Additionally the direct influence of the inhomogeneities on radiation transfer may generally destroy the $n=3$ behaviour of the SMS. Then the post-Newtonian instability cannot occur.

As a bottom line we find, that the interaction of the stellar component and the SMS can give rise to a secular evolution away from the unstable zero-energy state to a more stable structure. Additionally mass segregation may lead to a decoupling of the core region resulting in a sequence of SMS, the smaller and more compact ones nested in the larger ones. In order to show, whether this kind of evolution can happen, we will do detailed semi-analytical model calculations.

\section{References}

Amaro-Seoane, P. \& Spurzem, R. 2001, MNRAS, 327, 995

Fuller, G.M., Woosley, S.E., Weaver, T.A., ApJ, 307, 675

Hara, T. 1978, Progress of Th. Physics, 60, 711

Just, A., Kegel, W.H. \& Deiss, B.M. 1986, A\&A, 164, 337

Langbein, T., Spurzem, R., Fricke, K.J., Yorke, H.W. 1990, A\&A, 227, 333

Rees, M.J. 1984, ARA\&A, 22, 471 